\title{
Electrical Discharge Mitigation Strategies for Future CMS GEM Systems GE2/1 and ME0
}

\author{
Elizabeth Rose Starling* \\ on behalf of the CMS Muon Group \\ *Université libre de Bruxelles, Avenue Franklin Roosevelt 50, 1050 Bruxelles, Belgium \\ E-mail: elizabeth.rose.starling@cern.ch
}

In 2019-2020, the first of the CMS gas electron multiplier (GEM) systems, GE1/1, was installed into the CMS muon endcaps, to be fully operational by Run 3. This represents the first of three major GEM-based additions into CMS, to be followed in future runs by GE2/1 and the very forward muon tagger ME0. R\&D for these two future systems is currently well under way, with a focus on eliminating potential damage due to propagating electrical discharges within the detector, as was seen in the demonstrator system for GE1/1. This contribution presents results from the various mitigation strategies, including changes to the front-end readout electronics and to the construction of the detectors themselves. These results detail the reduction in propagating discharges from the various strategies, as well as unintended consequences of those strategies, such as the presence of bipolar cross-talk signals in chambers equipped with double-segmented GEM foils. Future prospects for the two systems will be discussed.

40th International Conference on High Energy physics - ICHEP2020

July 28 - August 6, 2020

Prague, Czech Republic (virtual meeting) 


\section{Introduction}

In preparation for the High Luminosity Large Hadron Collider (HL-LHC) at CERN, a series of upgrades are being made to the Compact Muon Solenoid (CMS) detector. This includes a set of three new muon subdetector systems, called GE1/1, GE2/1, and ME0, which are based on triple gas electron multiplier (triple-GEM) technology. Details on GE1/1 can be found in Reference [1] and details on GE2/1 and ME0 can be found in Reference [2].

Triple-GEMs are a subclass of micropattern gas detectors which operate on the principle of electron multiplication through cascading Townsend avalanches. Their primary feature is the presence of GEM foils, $50 \mu \mathrm{m}$-thick polyimide sheets that are coated with $5 \mu \mathrm{m}$ of copper on each side and etched with tapered holes (diameter of $70-140 \mu \mathrm{m}$, pitch of $50 \mu \mathrm{m}$ ) using a technique of masked photolithography. These layers are stacked on top of a PCB readout and sealed within a gas-tight volume that is flooded with a gas mixture of $70 \% \mathrm{Ar}$ and $30 \% \mathrm{CO}_{2}$. A high voltage is applied to the foils, which creates electric fields between the foils and through their holes. When a primary ionizing particle such as a muon passes through the active gas volume, the ionized electrons encounter these fields and multiply at each foil layer, for a total gain of $\sim 10^{4}$ at the readout board.

During the two-year (2017 - 2018) lifetime of the first CMS GEM demonstrator system, called the "slice test", a significant percentage of the front-end readout channels were lost as a result of propagating electrical discharges within the detectors. The primary discharge occurs first on the GEM foil closest to the anode, G3, as this is the only location within the GEM stack where the Raether limit of $\sim 10^{8}$ electrons can be exceeded and thus have a high enough local charge density to create a discharge. From here, the discharge may propagate either forwards or backwards within the GEM foil stack. If it propagates forwards from G3, it reaches the readout board, where the energy from the discharge can damage the delicate front-end readout electronics located there. This damage manifests itself as the channel loss seen during the slice test. If, instead, it propagates backwards before moving forwards towards the readout board, it gains energy at each backwards and forwards propagation, making the final jump to the readout board more likely to cause greater damage to the electronics. Given enough energy, the propagation can also reignite.

In order to preserve the lifetime of the full, final systems, a series of mitigation strategies have been employed in order to protect the sensitive front-end readout electronics against discharge damage, and minimize the number of discharges which occur in the first place. Following the successful installation of GE1/1 with its associated protection methods during Long Shutdown II, the focus of the discharge mitigation R\&D has shifted to GE2/1 and ME0. Two of the strategies which are being investigated for these systems will be discussed in this paper. Note that this is currently a very active area of research, and therefore the results presented herein may be preliminary.

\section{Double Segmentation}

In GE1/1 detectors, the surface of the GEM foil which faces the readout board is a single continuous conductor, whereas the other surface, which faces the drift board, is segmented into sectors, each of which are separated from each other and connected to the HV filter via a $10 \mathrm{M} \Omega$ protection resistor which is soldered directly onto the foil. Each of these sectors has an area of less 
than $100 \mathrm{~cm}^{2}$ by design, imitating the area of a $10 \times 10 \mathrm{~cm}^{2}$ detector. This segmentation and the corresponding HV filters are illustrated in Figure 1.

However, by leaving the bottom of the foil to act as a single continuous conductor with a high total capacitance, a higher probability for discharge propagation remains, as the energy stored here can be used to feed and even reignite the propagating discharge. To lessen this probability further, the bottom of the foil can be segmented as well, also into sectors of an area less than $100 \mathrm{~cm}^{2}$. Unlike the traditionally-segmented top

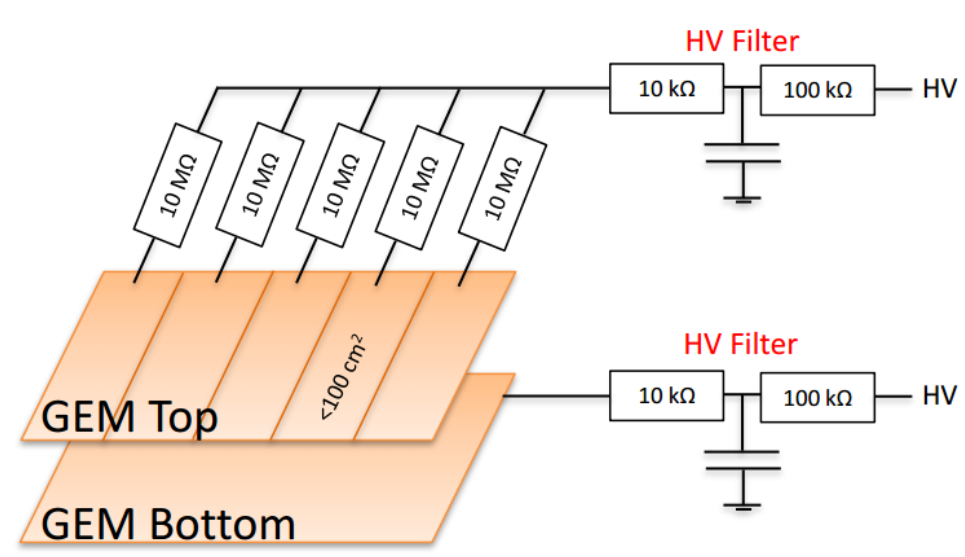

Figure 1: Illustration of a single-segmented GE1/1 GEM foil of the foil, the resistors present between the bottom segments are not protection resistors, but rather serve as decoupling resistors. In this way, not only is the capacitance of the foil reduced, but the HV sector decoupling is improved. Although this is still under study, a generally-acceptable value for these decoupling resistors has been found to be $100 \mathrm{k} \Omega$. In this way, a large ratio is maintained with the top protection resistors, and the bottom potential will not jump towards the top and potentially create a discharge propagation.

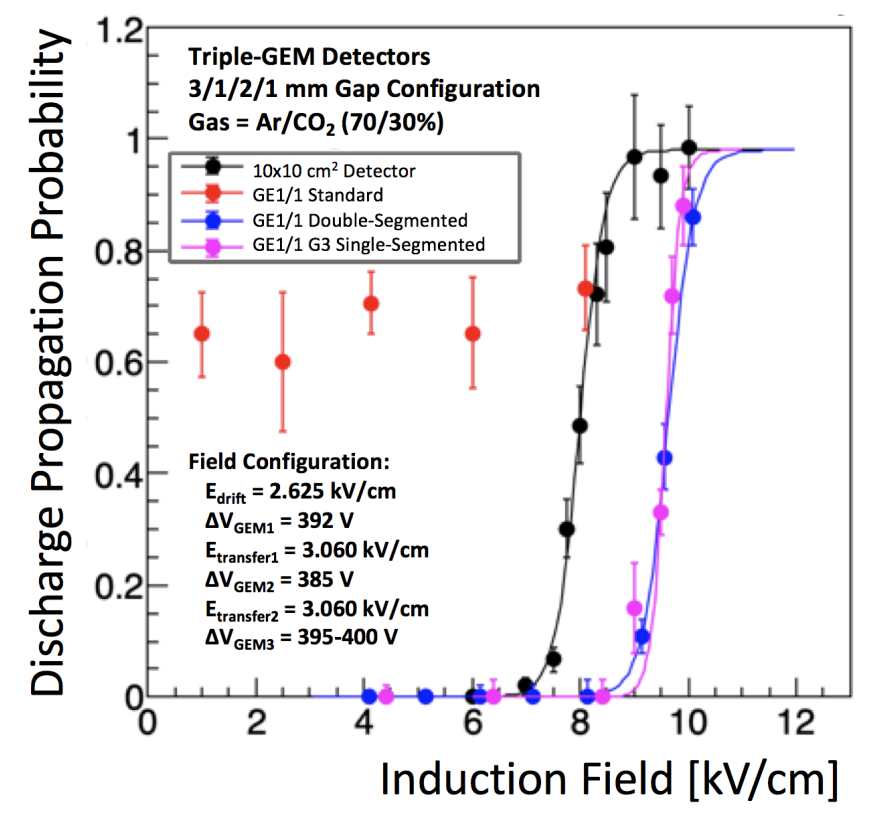

Figure 2: Probability of a propagating discharge by induction field for four GEM detector types.
Tests have shown this double segmentation to be extremely effective at reducing the probability of a propagating discharge occurring, as represented by the blue data in Figure 2. In these preliminary results, we see that the double segmentation provides a clear benefit against standard single segmentation, showing a lower probability of discharge propagation for induction fields up to $10 \mathrm{kV} \mathrm{cm}^{-1}$ than even a simple $10 \times 10 \mathrm{~cm}^{2}$ detector. Although more precision could most certainly be achieved in regards to the upper limit of discharge propagation probability, the data on the lower end of the induction field scale is clear. Indeed, at the nominal induction field present at CMS, $4 \mathrm{kV} \mathrm{cm}^{-1}$, no discharge propagation whatsoever was observed, in comparison to the $70 \%$ probability observed in a standard chamber. 
However, this brings with it an unexpected side-effect: a parasitic cross-talk signal. For an HV partition of an area on the order of $100 \mathrm{~cm}^{2}$, the low capacitance of the partition means that electrical potential fluctuations are propagated along the entirety of the partition. As a result, we see the propagation of true signals from a given readout sector in the adjacent readout sectors as a mirrored cross-talk signal via capacitive coupling.

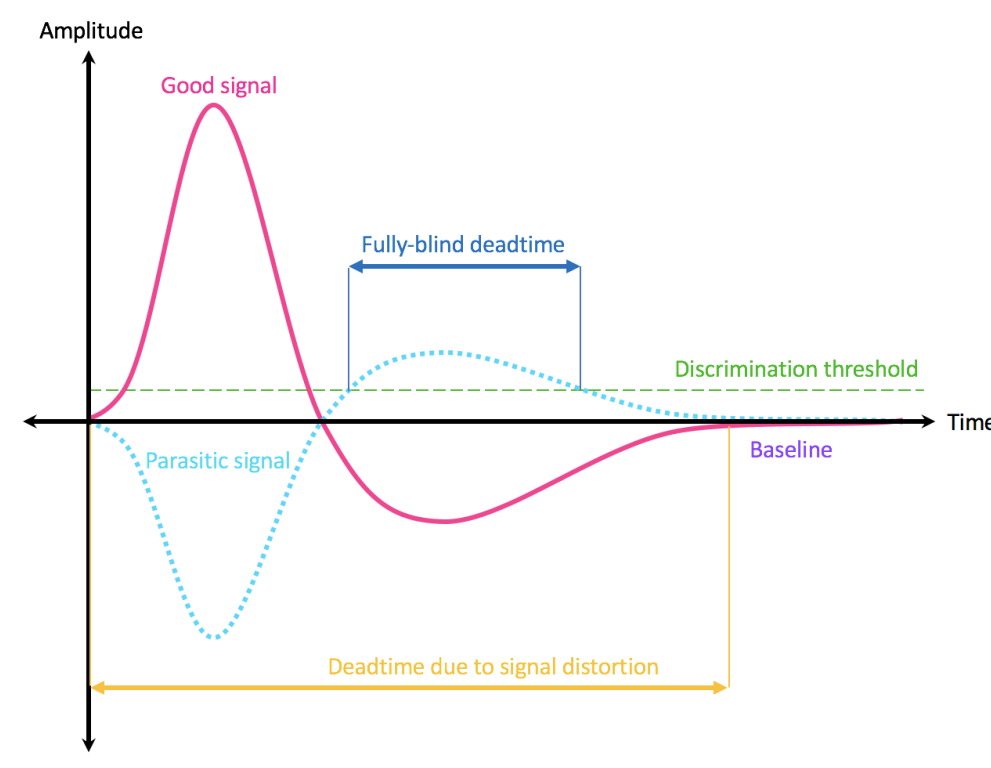

Figure 3: Example of a signal being mirrored in adjacent regions of a double-segmented GEM as seen from the analog output of the VFAT3 chip's preamplifier, and the deadtime that cross-talk signal induces

The problem, then, comes from the shape of the VFAT3's analog signals. The analog output signals of the VFAT3 chip's preamplifier are of dual polarity, consisting of a large initial portion of one polarity followed by a smaller portion of the opposite polarity. This shape is shown in Figure 3. The solid line represents the true signal that would be seen in the readout sector directly under radiation by the source. In order to detect this signals without being overwhelmed by noise, a discrimination threshold is set in the electronics, shown in the figure as the green dashed line. However, the signal's negative-polarity tail becomes positive when reflected in the mirror region of the neighboring readout sectors. In cases where the amplitude of the signal tail is high enough, as it is for HIPs, the reflected tail, which is now the same polarity as the main portion of the true signal, can exceed the discrimination threshold and falsely trigger the electronics as if it were also true signal. This then becomes the cross-talk signal which poses a significant threat to detector operations.

When the cross-talk signal is above the discrimination threshold, the VFAT is completely blind to new signal - no new signal, regardless of amplitude, can be detected at this time. However, we must consider the deadtime induced by the cross-talk signal to be the entire length of the signal, from the time it first appears until the time it returns to the baseline. If, for example, a minimally-ionizing particle was to be detected during the same time as the initial negative-polarity part of the cross-talk signal, even if it were to pass the discrimination threshold, the cross-talk signal would distort it to the point of it becoming unusable. It would be delayed in time from its true arrival time such that it would be impossible to assign it to the correct bunch crossing, and therefore it would be useless for particle reconstruction. In general, the maximum time it takes for the VFAT to return to a state in which it can detect a new signal is $500 \mathrm{~ns}$, equivalent to 20 LHC bunch crossings. This undesirably reduces the efficiency of the detectors, with preliminary studies by Piet Verwilligen and Jeremie A. Merlin showing GE2/1 to have up to a 3\% deadtime as a result.

Since a high capacitance results in a higher probability of discharge propagation, but a lower capacitance results in these signals, a study was performed to see if a middle ground could be found. 
A double-segmented GE1/1 detector was modified by replacing several of the $100 \mathrm{k} \Omega$ decoupling resistors at the narrow end of the bottom foil with $0 \Omega$ resistors, to mimic a single, larger HV partition of $\backsim 900 \mathrm{~cm}^{2}$. A baseline S-bit threshold scan was taken of the three VFATs at the narrow end of the detector: VFATs \#0, 8, and 16. Then a ${ }^{241} \mathrm{Am}$ source was placed under VFAT \#0 and a second S-bit threshold scan was taken. These were then compared to the same two scans taken with an unmodified double-segmented GE1/1 detector.
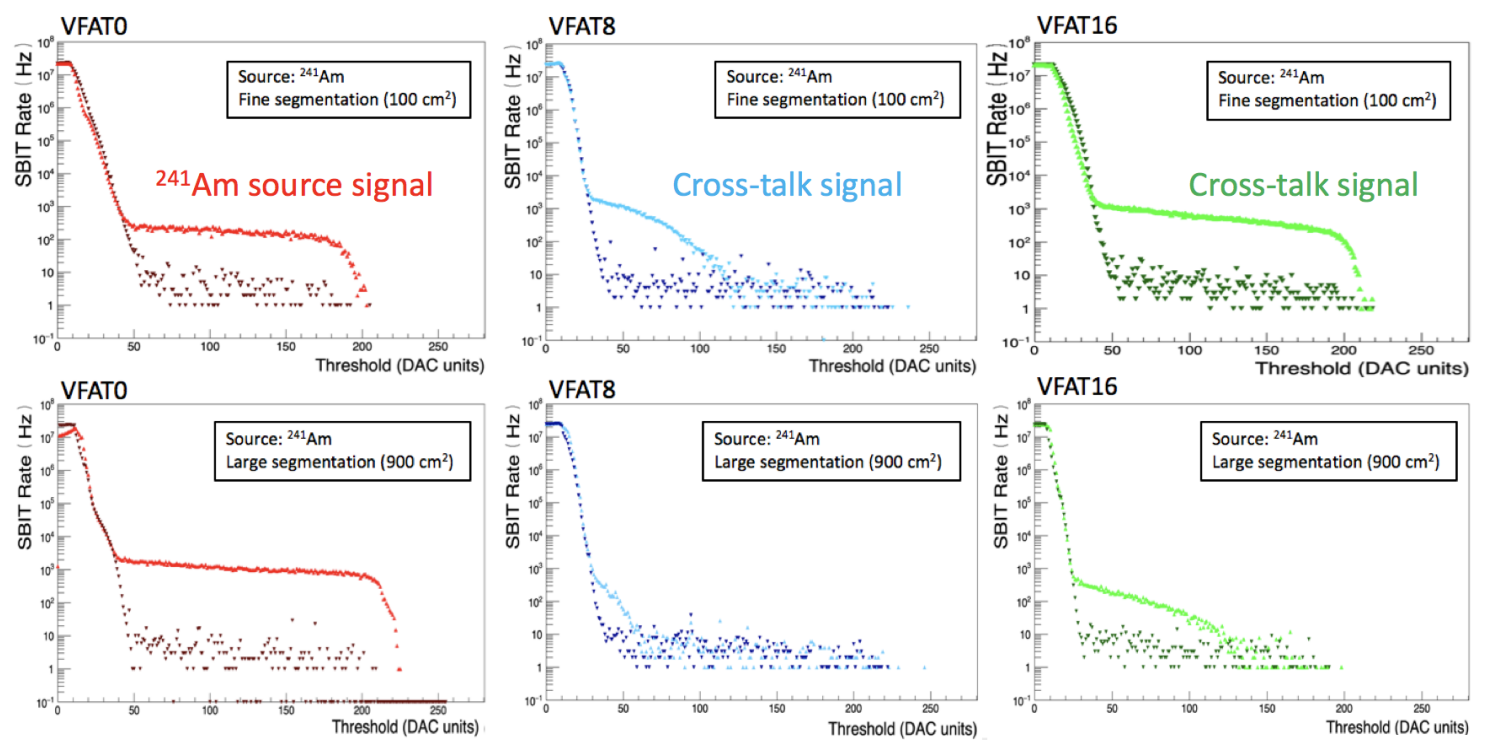

Figure 4: S-bit threshold scans for VFATs \#0, 8, and 16 for an unmodified and modified double-segmented GE1/1 detector. The darker colors represent the baseline scan, and the lighter colors represent the scan taken in the presence of an ${ }^{241} \mathrm{Am}$ source at VFAT \#0.

Figure 4 shows the S-bit threshold scans for the unmodified detector on top and the modified detector on the bottom. From this, we see that by enlarging the size of the HV partition from 100 $\mathrm{cm}^{2}$ to $900 \mathrm{~cm}^{2}$, the maximum amplitude of the cross-talk signal is decreased by a factor of two. However, the cross-talk signal is still present, and increasing the size of the HV partition any further would result in the problem returning to the original one of a large capacitance providing an energy store to the discharges. Thus, another solution is required.

One alternative solution is a partial double segmentation. Rather than segment the entire detector, only the first two foils would be double-segmented. In order to save on manufacturing costs, all three foils would be produced with the same mask, and, like in the study above, the bottom of G3 would have each HV sector connected to the next using $0 \Omega$ resistors to mimic one solid plane. This would still limit the overall energy available to the discharges reducing the problematic cross-talk signal by a factor of 30, making it essentially negligible. The simulation studies of Figure 5 show that with this configuration, the efficiency of the detector is brought nearly to the rate of having no cross-talk at all. Tests were performed using this alternative set-up in order to determine the propagation probability, with the results shown in pink in Figure 2. Here, we see that this new configuration's discharge probability curve closely resembles that of the fully double-segmented chamber, albeit with a steeper slope, presenting a very promising solution. 


\section{Additional Protective Measures}

Another method of lowering the probability of discharge propagation is the insertion of a drain resistor between the readout strips and the ground plane of the detector. Here, the precursor current which occurs between the initial discharge and the propagation of the discharge runs through the drain resistor rather than the readout electronics. Then the voltage drop across the drain resistor induces a drop in the potential of the strip, thereby temporarily suppressing the induction field. This prevents the discharge from propagating across the induction gap, protecting the front-end electronics, with a drain resis-

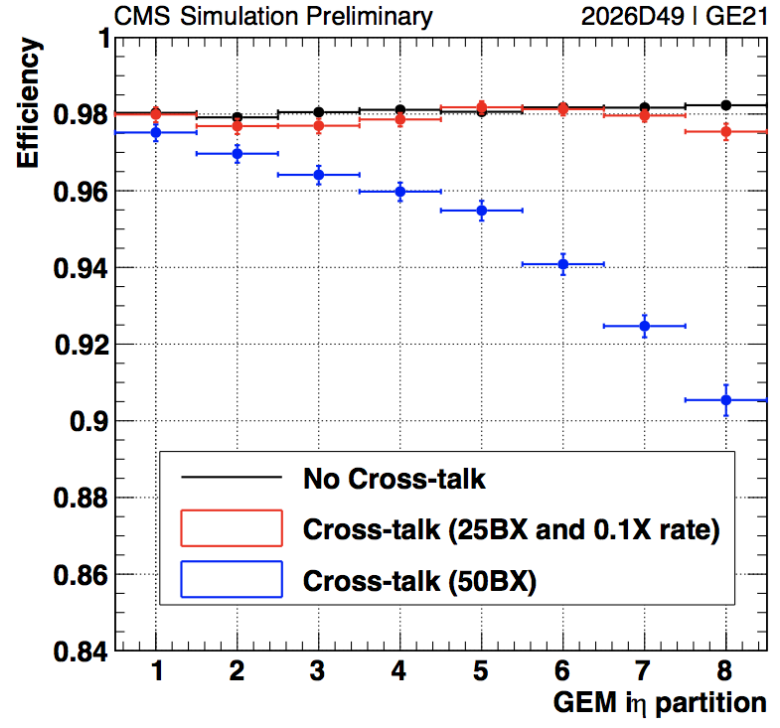

Figure 5: Efficiency of semi- and fully-double-segmented GE2/1 detectors according to the presence of cross-talk. tance as low as $100 \mathrm{k} \Omega$ reducing the propagation probability by a factor of more than $10^{3}$.

Finally, while the above two solutions lower the probability of a propagating discharge occurring, they do nothing to protect the front-end electronics in the event a propagating discharge does occur. For this, we turn to the use of protection circuits in front of the VFAT3 chip's input channels. As the choices for GE1/1 were limited by the size of the VFAT hybrid, the front-end card for GE2/1 has been redesigned to have a larger surface area to accommodate more robust protection circuits.

Three types of protection circuits are currently under consideration: a resistor-only circuit as is used in GE1/1, a resistor-and-capacitor circuit which would additionally prevent discharges from propagating, and a resistor-and-diode circuit which would prevent damage in the event of a discharge propagation. Tests of each circuit type's robustness against discharges and their effects on the efficiency of the detector are currently ongoing, with preliminary results showing promise, with robust protection and little effect on detector efficiency. Further testing is still required, however, for the final choice of protection circuit to be validated. Nevertheless, this represents a great step forward towards a long and healthy lifetime for the future GEM systems of CMS.

\section{Summary}

The R\&D campaign to eliminate discharge-related damage within future CMS GEM systems has thus far been quite successful, with a number of successful mitigation strategies proposed and implemented. Testing is still in progress, but we expect a long and healthy lifespan for both systems.

\section{References}

[1] CMS Collaboration (2015) CMS Technical Design Report for the Muon Endcap GEM Upgrade, Tech. Rep., [CERN-LHCC-2015-012 ; CMS-TDR-013].

[2] CMS Collaboration (2017) The Phase-2 Upgrade of the CMS Muon Detectors, Tech. Rep., [CERN-LHCC-2017-012; CMS-TDR-016]. 